\title{
Cervical Length, Volume and Flow Indices During Pregnancy by Transvaginal 2D and 3D Ultrasonography
}

\author{
${ }^{1}$ Alin Başgül Yiğiter, ${ }^{2}$ Zehra Neşe Kavak \\ ${ }^{1}$ Associate Professor, Chief, Bilim University School of Medicine, Department of Obstetrics and Gynecology, Fetal Maternal \\ Medicine Unit, Istanbul, Turkey \\ ${ }^{2}$ Professor, Marmara University School of Medicine, Department of Obstetrics and Gynecology, Fetal Medicine Unit
}

Correspondence: Alin Basgul Yiğiter, Associate Professor, Chief, Department of Obstetrics and Gynecology, Fetal Maternal Medicine Unit, Abide-i Hürriyet Caddesi, No: 144, Kat: 3, D:10, 34381, Şişli, Ístanbul, Turkey, Phone: 05065944463 Fax: +90 21221958 29, e-mail: alinbasgul@hotmail.com

\begin{abstract}
Recently, many researches suggested that 2D US is insufficient for defining the true sagittal plane of uterine cervix. When the cervix is bent or curved, it is difficult to get a perfect sagittal section but rather mid oblique image by 2D US. Only 3D US allows us to evaluate the cervix in the coronal section and reproduce images in many cut. Coronal section of the cervix is especially useful in evaluating cervical funneling. Diagnosing internal orifice dilatation, asymmetrical, flattened or fissure-like cervices is possible. 3D US favors a more detailed study of cervical anatomy and biometry than 2D US. Cervical evaluation by 3D multiplanar sections can be added in the screening for preterm labor.

Three-dimensional (3D) imaging combined with power Doppler, theoretically provides the possibility to assess the volume and quantify the power Doppler signal in the whole target organ, whereas, information from 2D US on vascularization and blood flow is restricted to a single subjectively chosen $2 \mathrm{D}$ plane. The measurement of cervical indices is reproducible and may be used in clinical practice and research to determine the changes of the cervical morphology and vascularization in pregnancy. Consequently, further studies of 3D ultrasound imaging of the cervix in pregnancy and clinical correlations to obstetrical events are required for better understanding the physiology and functional pathophysiology of the cervix during pregnancy.
\end{abstract}

Keywords: Three-dimensional ultrasound, VOCAL, cervical length measurement, volume, power Doppler.

\section{Why is It Important to Evaluate the Cervix During Pregnancy?}

Prematurity is a major cause of perinatal morbidity and mortality despite improved postnatal care. ${ }^{1}$ Consequently, identification of risk factors and early cervical changes associated with preterm birth is an important area of investigation. $^{2}$

\section{Cervical Length Assessment Methods in Pregnancy}

There are different ways of evaluating cervix during pregnancy. As the cervix begins to dilate from the internal os, the standard digital examination of cervix is limited, subjective and unsatisfactory.

The measurement of cervical length can be done by transabdominal way but to visualize cervix with full bladder is required which lengthens the cervix and lessens the accuracy. $^{3}$
The translabial or transperineal US can fail sometimes to measure the cervix mainly due to bowel gas. ${ }^{4}$ The transvaginal approach has therefore become the accepted method for cervical length assessment.

Recently, transvaginal three-dimensional ultrasound has been used in evaluating uterine cervix. One can obtain uniquely multiplanar sectional planes of cervix. It is a method which is capable of obtaining simultaneous visualization of the three different imaging planes: sagittal, transverse and coronal (Fig. 1). A true coronal plane can be displayed by direct correlation of the views in a multiplanar display. By 3D US large volume of data can be acquisited and stored. One can reanalyze the data in different planes. 3D US being more accurate has greater diagnostic potential on cervical evaluation during pregnancy. $3,5,6$

\section{D US versus 2D US to Measure Cervical Length}

It has been suggested that 2D US is ineffective for defining the true sagittal plane of uterine cervix due to differences in 


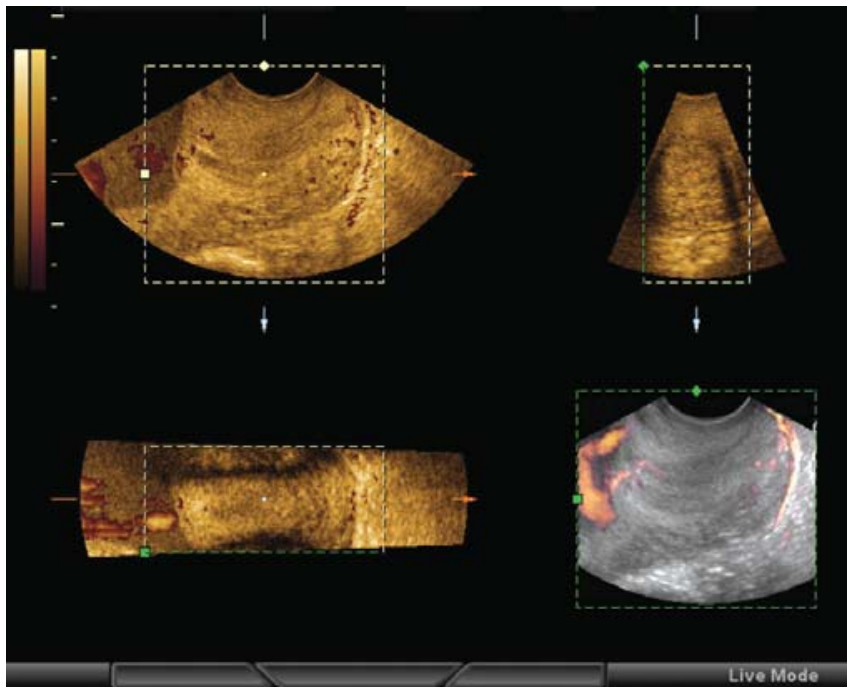

Fig. 1: A 3D multiplanar cervical image combined with power Doppler. Multiplanar display can visualize sagittal, axial and coronal planes simultaneously. The cervical volume assessment and quantification of the power Doppler signal in the whole target organ is possible

cervical length measurement results. ${ }^{5-7}$ Also, by 2D US evaluation of the cervical length is sometimes difficult for example, when, the cervix is bent or curved it is difficult to get a perfect sagittal section but rather mid oblique image by $2 \mathrm{D}$ US.

Only 3D US allows us to evaluate the cervix in the coronal section and reproduce images in many cut. Coronal section of the cervix is especially useful in evaluating cervical funneling or in cases for example in diagnosing internal orifice dilatation, asymmetrical, flattened or fissure-like cervices, that is why 3D US favors a more detailed study of cervical anatomy and biometry than 2D US. ${ }^{3,5,7,8}$

Severi et al found that 3D US measures the cervical length longer than the 2D US (39.8 mm versus $37.6 \mathrm{~mm}$ accordingly) in sagittal sections. ${ }^{5}$

They also found 3D US superior to 2D US regarding diagnosing internal cervical os dilatation by the capability of obtaining coronal sections and digital manupulation capacity allowing a reliable identification of the endocervical canal and internal orifice, resulting in more accurate cervical biometry. They explained this results as by 3D US a more correct, midsagittal plane can be determined and that is why 3D US is more accurate in cervical length measurement.

Bega et al compared the findings on 3D US with those on conventional two-dimensional ultrasound for 21 pregnant women at high-risk for premature delivery at 11 and 32 weeks' of gestation. ${ }^{7}$ They concluded that 3D US offer a more complete assessment of the cervix than 2D US and agreed that 3D US improves the understanding of cervical morphology.

Towner et al compared cervical length measurements obtained by transvaginal two-dimensional (2D) and transvaginal three-dimensional (3D) ultrasound. ${ }^{6}$ They examined 34 women with vaginal assessment of cervical length. The mean cervical 2D length was $35.7 \mathrm{~mm}$ and 3D length was $43.7 \mathrm{~mm}$. In all but one woman the 3D length obtained was the same or longer than the 2D length. They concluded that the longer the cervical length on 3D imaging, the greater the difference from the $2 \mathrm{D}$ length. They explained longer 3D cervical mesurements by; 2D US having difficulty to overcome the anatomical factors. Thinking that cervical legth measurement is used in predicting preterm delivery more accurate results for cervical legth by 3D US could improve predictive value of transvaginal ultrasound in assessing the risk for preterm delivery.

\section{The Anatomic and Technical Difficulties of 2D Transvaginal Cervical Evaluation}

Yost et al evaluated 60 high risk patients for preterm delivery by by transvaginal ultrasound for cervical evaluation. ${ }^{9}$ They encountered with undeveloped lower uterine segment, focal myometrial contraction, rapid and spontaneous cervical change and an endocervical polyp. Technical pitfalls included incorrect interpretation of internal os dilation because of vaginal probe orientation and artificial lengthening of the endocervical canal because of distortion of the cervix by the transducer.

\section{When It is Short Cervix?}

The mean cervical length is $35 \mathrm{~mm}$ to $40 \mathrm{~mm}$. A cervical length of $25 \mathrm{~mm}$ has generally been found to be at the 10th percentile ${ }^{3,10}$ (Fig. 2).

\section{D US versus 2D US to Calculate Cervical Volume and Blood Flow}

Measuring cervical volume can reflect cervical morphology better. Three-dimensional (3D) imaging combined with power Doppler, theoretically provides the possibility to assess the volume and quantify the power Doppler signal in the whole target organ (Fig. 1), whereas, information from 2D ultrasound on vascularization and blood flow is restricted to a single subjectively chosen 2D plane ${ }^{11,12}$ (Fig. 3).

There is evidence that angiogenic factors may play a role in cervical ripening and the birth process, therefore it 


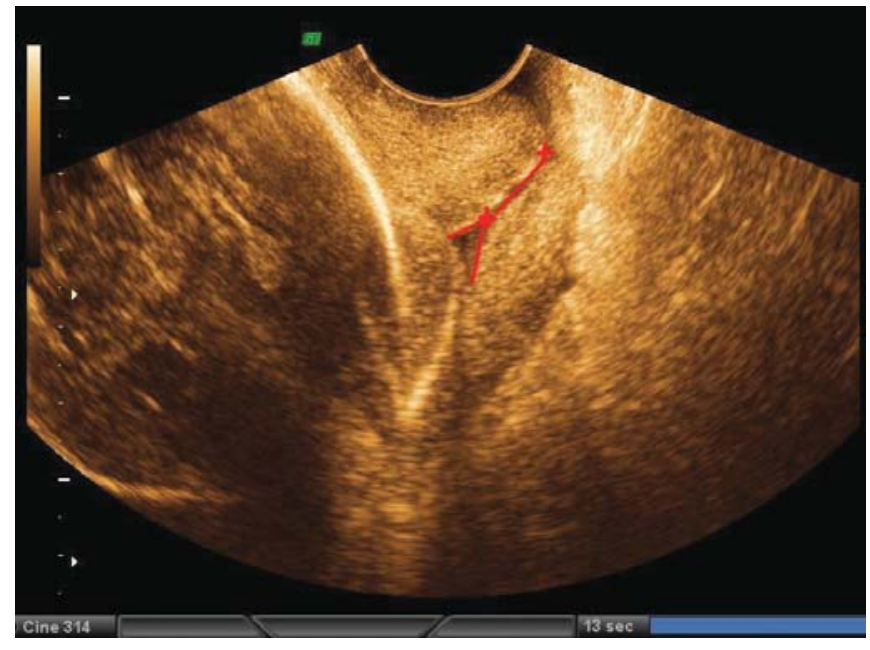

Fig. 2: A 2D sagittal view of the cervix in a patient with preterm delivery. The cervix is shortened and there is funneling through which the amniotic membrane is prolapsed

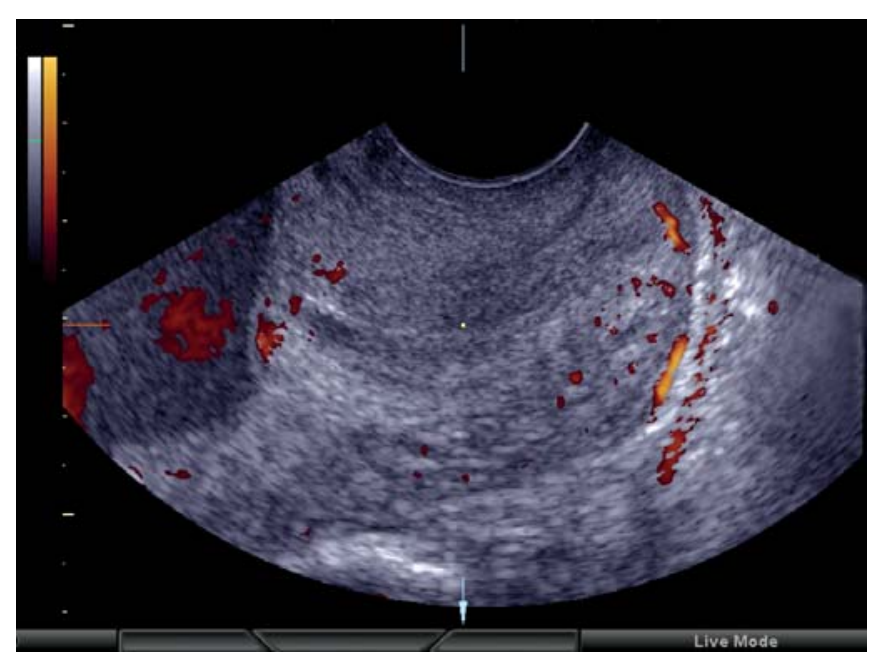

Fig. 3: A 2D cervical image combined with power Doppler. The information on vascularization and blood flow is restricted to a single subjectively chosen $2 \mathrm{D}$ plane

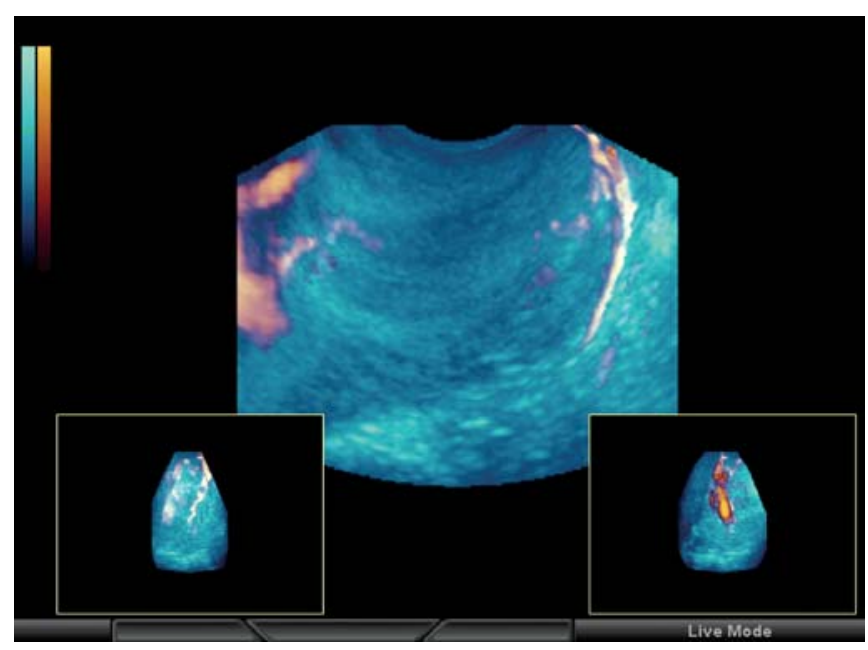

Fig. 4: A 3D US combined with power Doppler of the cervix in the 26th weeks of pregnancy using the glass body appearance mode would be valuable to know how cervical blood circulation changes during pregnancy. ${ }^{13}$

Imaging the cervix by 3-dimensional (3D) ultrasound provides a more accurate knowledge about the cervical morphology. ${ }^{14}$ 3D ultrasonography has the potential to provide more accurate volume measurements than does conventional 2-dimensional (2D) ultrasound. ${ }^{11}$ The ability to save and store volumes of US data for later analysis is also of great advantage and opens up new possibilities for clinical networking and consultation. ${ }^{11-13}$

\section{What is the Power Doppler Angiography (PD) and the Virtual Organ Computer-aided Analysis Imaging Program (VOCAL) for Cervical \\ Evaluation?}

One of the latest technical achievements in the field of ultrasonography is 3D imaging combined with power Doppler, which provides the potential to quantify power Doppler signals in a whole organ (Fig. 4). ${ }^{13}$

Few studies are available to describe the application of transvaginal 3D ultrasonography, Power Doppler angiography (PD) and The Virtual Organ Computer-aided Analysis imaging program (VOCAL) for cervical evaluation. ${ }^{13-16}$

VOCAL is a software, which is integrated into the 3D ultrasound system, which is used to calculate cervical volume $\left(\mathrm{cm}^{3}\right)$ and power Doppler flow indices from the orthogonal multiplanar views of the cervix obtained from the volume acquired (Figs $5 \mathrm{~A}$ and B). Using the histogram facility of the VOCAL software the following Doppler indices can be calculated: Vascularization index (VI), flow index (FI) and vascularization flow index (VFI).

\section{What is the Technique to Calculate the Cervical Volume and Vascularization Indices with 3D US VOCAL Technique?}

The patient must be examined in the lithotomy position with an empty bladder.

The ultrasound probe must be slowly introduced into the vagina and care must be taken to avoid under pressure on the cervix. After a satisfactory gray-scale image of the cervix had been obtained, the probe can be withdrawn until the image become blurred. Then the probe can be gradually advanced again with only enough pressure to restore a satisfactory image. A sagittal view of the cervix where, the internal os, the cervical canal and the external os were, all 

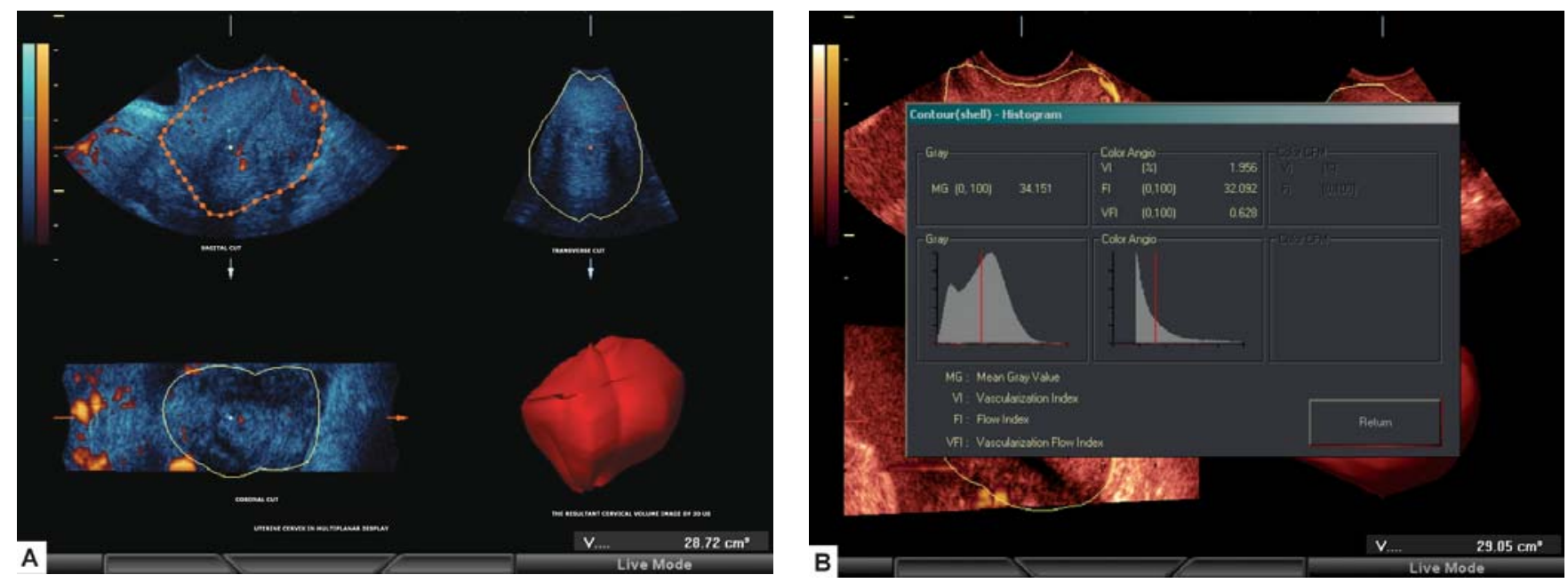

Figs 5A and B: (A) A 3D ultrasound measurement of cervical volume by VOCAL. (B) Multiplanar cervical images: upper left (the cervix is shown demarcated by lines), upper right and lower left quadrants show longitudinal, transverse and coronal planes of the cervix respectively. Lower right image shows the resultant 3D image of the cervix

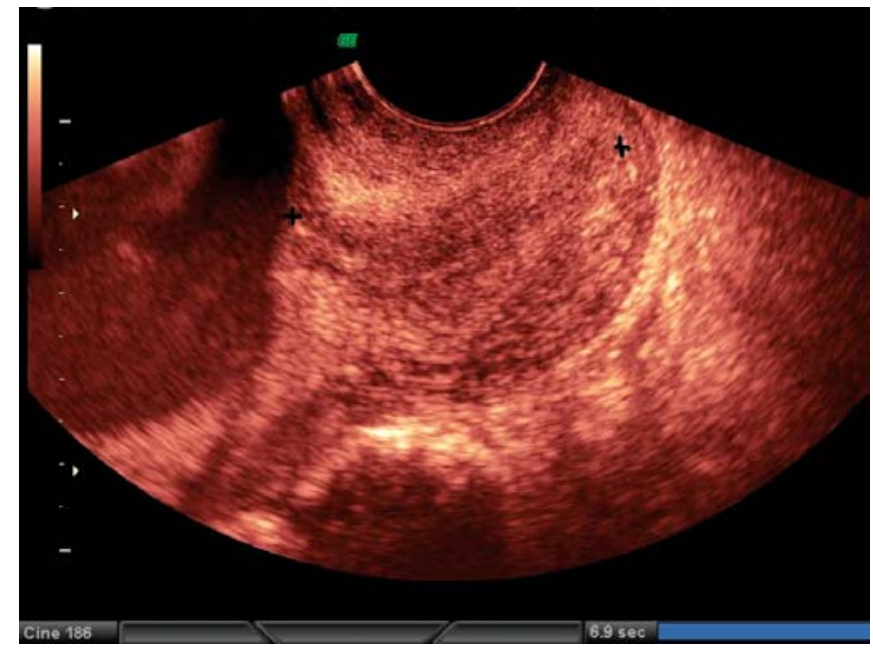

Fig. 6: A 2D US sagittal view of the cervix in the third trimester of the pregnancy. The internal and external cervical os is marked by the plus sign

seen at the same time must be obtained (Fig. 6). The system can then be switched into the power Doppler mode and then into the 3D mode. The cervix must be centralized within the 3D sector appearing on the ultrasound screen, and data can be obtained by holding the transducer stationary while its crystals were mechanically rotated across the sector with a sweep angle of $90^{\circ}$. The fast volume acquisition (low resolution) setting can be preferred to minimize periodic flashing artifacts arising from pulsation of the uterine arteries and from fetal movements. The duration of the volume acquisition is usually $15-20 \mathrm{~s}$ depending on the dimensions of the $3 \mathrm{D}$ sector. ${ }^{13-16}$
The scanned volumes can be stored digitally for analysis off-line. Calculation of the surface geometry is the first step of VOCAL and is defined by rotating an image plane around a fixed axis (the main contour axis) and defining 2D contours on each plane. The contour mode in the VOCAL program was then set to manual.

Drawing must be started and continued from the same chosen section through the cervix namely "reference image". The rotation steps can be $6^{\circ}, 9^{\circ}, 15^{\circ}, 30^{\circ}$. For example, if you choose rotation steps as $30^{\circ}$, you need to draw the contours of the cervix six times manually using the roller ball cursor of the system or with your fingers on the digital screen.

Once the contour of the cervix is defined, the VOCAL program automatically calculates the volume and power Doppler vascularization index, flow index and vascularization-flow index.

\section{What is the Technique to Calculate the Cervical Length by 3D US Multiplanar Section}

After the volume acquisition imagesare simultaneously displayed on the monitor in an orthogonalplane (multiplanar display) in axial, transverse and coronal cuts, it is possible to manipulate digitally and reconstruct the desired images. ${ }^{7,8}$

\section{Is There Any Major Change in Cervical Volume During the Pregnancy Calculated by 3D US?}

A recent study resulted that there is no statistically significant change in cervical volume with gestational age. However, 


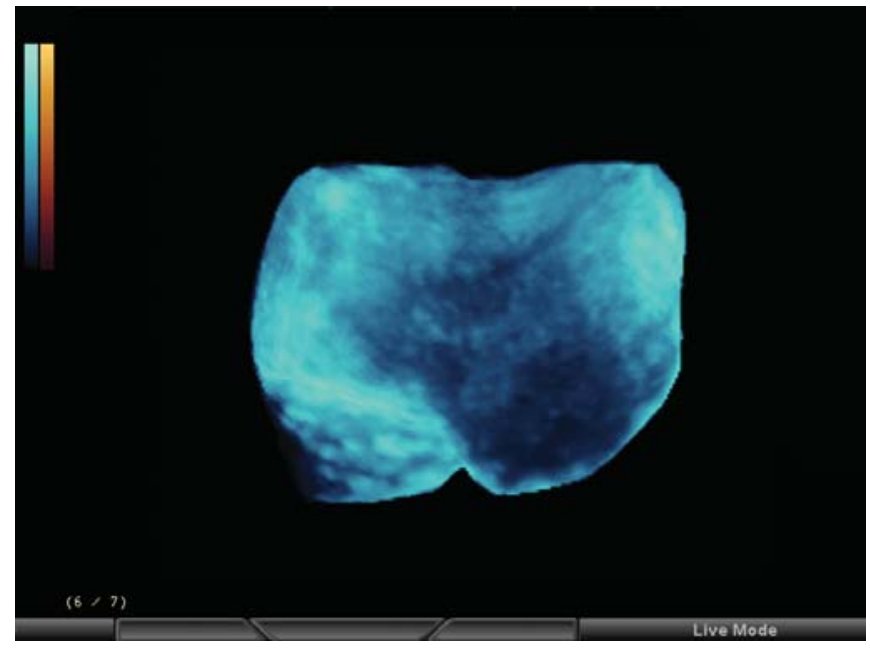

Fig. 7: The resultant 3D volume image of the cervix by glass body appearance mode

cervical volume was found to be slightly smaller at 41 weeks than it was at 17-40 weeks' gestation. ${ }^{13}$ Cervical flow indices also did not change. However, they noted that this does not exclude the possibility of too small vascular changes which is not detectable with the rather crude Doppler ultrasound technique.

\section{Why is It Difficult to Measure the Cervical Volume?}

Although volume is a common unit for classification and comparison, some authors suggest that this method seems difficult for the cervix beceause the sonographic demarcation between the cervix, the lower uterine segment and the surrounding vaginal tissue is not clear (Fig. 7). ${ }^{13-16}$

\section{Does Parity Effects Cervical Volume and the Vascular Indices Measured by 3D US?}

There are only few studies comparing the cervical volumes and the blood flow regarding the parity. Recent two studies revealed no statistically significant differences between nulliparous and parous women. , $^{13,16}$

Our results suggest that parity does not effect the cervical volume and flow indices in the pregnant women. Although there are individual differences, the cervix of parous women decreases in length and increases in width from midpregnancy to term. ${ }^{16}$ However, our study group was small and additional studies on cervical volume and flow indices associated with gestational age, parity and prepregnancy BMI should be undertaken with a larger sample to determine the normal values and cut-off points for prediction of preterm delivery in pregnant women.

\section{CONCLUSION}

As 3 US increase in the accuracy of the cervical length measurements in pregnant women cervical evaluation by 3D multiplanar sections can be added in the screening for preterm labor. However, there is a need to study a higher number of cases to establish criteria for measurement of the cervical length and further researches with a higher number of cases are necessary for confirming these results. ${ }^{3,6,17}$

Recent researches suggest that the measurements of cervical indices are reproducible and may be used in clinical practice and research to determine the changes of the cervical morphology and vascularization in pregnancy. These changes appear to be important to understanding physiological reactions of the cervix to term labor as well as pathological processes in the cervix that preceede preterm labor. In addition, this information may clarify the sonographic counterparts of the clearly anatomical changes observed after birth and characterize the parous cervix. ${ }^{13-16}$ At current stage of knowledge, however, volume and vascularity assessment of the cervix should be considered experimental and further studies of 3D ultrasound of the cervix in pregnancy are required to assess the potential of these sophisticated imaging methods.

\section{REFERENCES}

1. Andersen HF, Nugent CE, Wanty SD, et al. Prediction of risk for preterm delivery by ultrasonographic measurement of cervical length. Am J Obstet Gynecol 1990;163:859-67.

2. Matijecic R, Vasilj O, Grgic O. Ultrasound based diagnosis of cervical insufficiency and evidence to support the intervention. In: Kurjak A, Chervenak FA, editors. Donald school textbook of Ultrasound in Obstetrics and Gynecology, 2nd ed. India: Jaypee brothers medical publishers 2008;465-72.

3. Brandaol SR, Murta CGV, Moron AF,Mattar R, Pires CR,Guerzet EA. Three-dimensional ultrasound of uterine cervix in pregnancy: Prospects. Radiol Bras 2006;39:4:305-08.

4. Maymon R, Herman A, Ariely S, Dreazen E, Buckovsky I, Weinraub Z. Three-dimensional vaginal sonography in obstetrics and gynecology. Human Reproduction 2000;6:475-84.

5. Severi MF, Bocchi C, Florio P, Picciolini E, D’Aniello G, et al. Comparison of two-dimensional and three-dimensional ultrasound in the assessment of the cervix to predict preterm delivery. Ultrasound Med Biol 2003;29:1261-65.

6. Towner D, Boe N, Lou K, Gilbert M. Cervical length measurements in pregnancy are longer when measured with threedimensional transvaginal ultrasound. J Matern Fetal Neonatal Med 2004;16:167-70.

7. Bega G, Lev-Toaff A, Kuhlman K. Three-dimensional multiplanar transvaginal ultrasound of the cervix in pregnancy. Ultrasound Obstet Gynecol 2000;16:351-58. 
8. Blaas HG, Eik-Nes SH, Berg S. Three-dimensional fetal ultrasound. Baillieres Best Pract Res Clin Obstet Gynaecol 2000;14:611-27.

9. Yost NP, Bloom SL, Twickler DM, Leveno KJ. Pitfalls in ultrasonic cervical length measurement for predicting preterm birth.Obstet Gynecol 1999;93:510-16.

10. Strauss A, Heer I, Fuchshuber S, Janssen U, Hillemanns P, et al. Sonographic cervical volumetry in higher order multiple gestation. Fetal Diagn Ther 2001;16:346-53.

11. Kurjak A. 3D ultrasound and perinatal medicine. J Perinat Med 2002;30:5-7.

12. Kurjak A, Merce LT, Badreldeen A. 3D Power Doppler in the study of fetal and maternal angiogenesis and vasculogenesis. In: Kurjak A, Chervenak FA, editors. Donald school textbook of Ultrasound in Obstetrics and Gynecology, 2nd ed. India: Jaypee brothers medical publishers 2008;433-51.

13. Rovas L, Sladkevicius P, Strobel E, et al. Reference data representative of normal findings at three-dimensional power Doppler ultrasound examination of the cervix from 17 to 41 gestational weeks. Ultrasound Obstet Gynecol 2006;28(6): 761-67.

14. Basgul A, Kavak ZN, Bakirci N, et al. Intra- and interobserver agreement on cervical volume and flow indices during pregnancy using transvaginal 3-dimensional ultrasonography and Doppler angiography. Int J Fertil Womens Med 2006;51(6):256-61.

15. Rovas L, Sladkevicius P, Strobel E, et al. Intraobserver and interobserver reproducibility of three-dimensional gray-scale and power Doppler ultrasound examinations of the cervix in pregnant women. Ultrasound Obstet Gynecol 2005;26:132-307.

16. Basgul A, Kavak ZN, Bakirci N, et al. Three-dimensional ultrasound power Doppler assessment of the cervix: comparison between nulliparas and multiparas. J Perinat Med 2007;35(1):4850 .

17. Rozenberg P, Rafii A, Senat MV, Dujardin A, Rapon J, et al. Predictive value of two-dimensional and three-dimensional multiplanar ultrasound evaluation of the cervix in preterm labor. J Matern Fetal Neonatal Med 2003;13:237-41. 